\section{ENRICO FERMI}

\section{Enrico Fermi}

The Man and his Theories. By Pierre de Latil. Translated by Len Ortzen. (Profiles in Science.) Pp. 178+ Illustrations. (London: Souvenir Press, Ltd., 1965.) $21 s$.

1 HIS is another book in a series of concise works each of which deals with the life and work of a scientist. Pierre de Latil tells the story of Fermi's life, with ample detail to bring the man to life without swamping the explanations of the scientist's achievements.

As a young Roman student, Fermi began studying when the first explanations of the new physics had gained acceptance. Ho was able to build on this work to such an extent that he participated in most of the following stages of the discovery of atomic energy. In particular, he was responsible for the design and construction of the first self-sustaining uranium-graphite pile set up underneath the grandstand of the squash court at Stagg Field, the University of Chicago Stadium, in 1942, and he was one of the chief figures in the making of the first atomic bomb.

In the first place he worked as a theoretician, but this led him to spend an ever-increasing time on experimental work. The lack of technical means for continuing his experiments forced him to become his own engineer. His ability in all three fields enabled him to tackle the vast problems which he considered. He could have re-organized the whole of Italian physics teaching, but the Dictatorship in his own country was no more tolerant and enlightened than that in Germany, and in 1938 he accepted an appointment at Columbia University, immediately after his award of a Nobel Prize, which actually assisted his escape from his native land. The United States, with its vast resources, suited his genius, but for a time he was regarded as an enemy alien and subjected to security control. Indeed, although he entered the United States with a Nobel Prize, he was subjected to an infantile examination in arithmetic. Fermi died philosophically and stoically from internal cancer at the age of fifty-three.

The translation of the book, though it projects the spirit of the original, is sometimes defective; for example, fluorine and nitrogen appear as fluor and azote, respectively. Some surnames, such as those of Wigner and Bethe, are misspelt.

The chronology of some of the events in the history of atomic physies is open to question. For example, the discovery of artificial radioactivity in 1934 is described as the "first time that man had provoked activities within the nucleus of the atom". Cockcroft and Waiton (1932) are not even mentioned, but the transmutation of the lithium atom might be regarded as a man-made nuclear activity. The discovery of radioactivity by Becquerel early in 1896 is described as "through pure instinct as an experimental physicist". It is surely well known that Becquerel read Röntgen's X-ray paper of December 1895, and this suggested to him an experiment of a complementary nature, which, while it did not give the expected result, quickly led to the discovery of the phenomenon of radioactivity.

This useful book is well produced and contains many good illustrations.

W. L. SUMNER

\section{WAVE PROPAGATION THROUGH THE IONOSPHERE}

Lectures on Modern Magneto-ionic Theory

By K. G. Buddən. (Documents on Modern Physics.) Pp. 82. (London wind Glasgow: Blackie and Son, Ltd., 1964.) $30 s$.

7 HE purpose of this book is to introduce advanced students to the theory of wave propagation through the ionosphere. This transmitting medium is both absorbing and doubly refracting, and it often varies with distance neither slowly nor quickly compared with a wavelength; an understanding of how waves travel through it therefore involves consideration of several fundamental ideas of considerable complexity. The book includes, for example, discussion of wave packets, group velocity, signal velocity and energy velocity, resolution into angular spectra, the relation between ray surfaces and refractive index surfaces, the impulse response of transfer functions, Sommerfeld precursor waves, reflexion coefficients and their relation to characteristic admittances, WKB solutions, phase integral methods, and the methods of steepest descent. Although the topics discussed are of immediate importance for ionospheric theory they are treated so generally that they can be applied immediately to the propagation of other kinds of waves.

Dr. Budden has not attempted to write a mathematical text-book; to some extent he assumes that the basic theory has been studied elsewhere and his aim is to discuss it for the purpose of undorstanding the physical behaviour of the medium and of the waves. His book will help the advanced student, for whom it is intended, to roalize that mathematics is not just a substitute for an automatic computer which provides the 'answer', but if it is followed intelligently step by step it also provides a precise description of the physical processes concerned. It is a guide rather than a text-book and, because it is based on a lecture course, it is ideally suited for its purpose. Dr. Budden, well known as a lecturer and a director of research stud $m$ ts, has performed a considerable service in making some of his bəst lectures available more widely in this way.

The book can be recommended to all those who work in the complicated subject of wave propagation through magneto-plasma. Beginniners will use it to help them to und irstand text-books and papers; established workers will use it to elarify their ideas; and experts will read it to admire the way in which the essentials of a problem are exposed in all their simplicity. Those who believe they have fully understood a problem when they have discussed it with the help of a complicated piece of mathematics will learn from this book how it is possible to acquire an even deeper und rrstanding through a detailed discussion of what the analysis means.

\section{J. A. Ratcliffee}

\section{CHEMICAL REACTIONS OF POLYMERS}

\section{Chemical Reactions of Polymers}

Edited by E. M. Fettes. (High Polymers: a Series of Monographs on the Chemistry, Physics, and Technology of High Polymeric Substances, Vol. 19.) Pp. xxii +1304. (New York and London: Interscience Publishers, a Division of John Wiley and Sons, 1964.) $300 s$.

CHEMICAL reactions of high polymers are of con1 siderable scientific and technological importance. Knowledge of reactions that may occur during processing, exposure to heat. light and other forms of radiation, and to oxygen or ozone, is essential to an understanding of the factors governing polymer stability in different environments. Technological applications of high polymers have been greatly extended by the use of such reactions as those causing cross-linking, the combination of different polymers and modification of structure and therefore of properties. Although many of these reactions have been known for a long time and, with others, have been extensively studied, the general subject of chemical reactions of polymers has not previously received separate treatment. This book, intended for graduates in chemistry and in which a knowledge of the structure and properties of polymers is assumed, is designed to give a critical survey of the various types of reaction in which at least one re- 